\title{
NANOPARTÍCULAS DE LIPÍDIOS SÓLIDOS: MÉTODOS CLÁSSICOS DE PRODUÇÃo LABORATORIAL
}

\section{Eliana B. Souto*}

Faculdade de Ciências da Saúde, Universidade Fernando Pessoa, 4200-150 Porto / Instituto de Biotecnologia e Bioengenharia, Centro de Genómica e Biotecnologia, Universidade de Trás-os-Montes e Alto Douro, 5001-801 Vila Real - Portugal

\section{Patrícia Severino}

Departamento de Processos Biotecnológicos, Faculdade de Engenharia Química, Universidade de Campinas, 13083-852 Campinas

- SP, Brasil / Faculdade de Ciências da Saúde, Universidade Fernando Pessoa, 4200-150 Porto - Portugal

Maria Helena A. Santana

Departamento de Processos Biotecnológicos, Faculdade de Engenharia Química, Universidade de Campinas, 13083-852 Campinas - SP, Brasil

\section{Samantha C. Pinho}

Departamento de Engenharia de Alimentos, Faculdade de Zootecnia e Engenharia de Alimentos, Universidade de São Paulo, 13635- 900 Pirassununga - SP, Brasil

Recebido em 7/1/11; aceito em 20/7/11; publicado na web em 2/9/11

\begin{abstract}
SOLID LIPID NANOPARTICLES: CLASSICAL METHODS OF LAB PRODUCTION. Solid lipid nanoparticles have been extensively investigated as drug delivery systems. These colloidal systems have major advantages compared to others more traditional. Reported advantages include sustained release, ability to solubilize lipophilic drugs, increased physical and chemical stability of labile molecules, decreased unwanted side-effects showing lower toxicity, and scale up facilities. This paper aims at reviewing the traditional methods of solid lipid nanoparticles production, such as fusion-emulsification (hot and cold), solvent evaporationemulsification and microemulsion, dealing with the main technological parameters that influence the quality properties of solid lipid nanoparticles.
\end{abstract}

Keywords: solid lipid nanoparticle; production of methods; technological parameters.

\section{INTRODUÇÃO}

Nanopartículas lipídicas sólidas (NLS) foram desenvolvidas em 1991, como um sistema alternativo de encapsulação de princípios ativos em relação aos sistemas coloidais tradicionais, tais como emulsões, lipossomas e nanopartículas poliméricas. O grande diferencial das NLS é a sua excelente estabilidade físico-química, que proporciona maior proteção contra a degradação de fármacos lábeis. ${ }^{1,2}$ $\mathrm{Na}$ área farmacêutica, as NLS podem ser usadas por todas as vias de administração, como a oral, ${ }^{3}$ parenteral ${ }^{4}$ e cutânea, ${ }^{5}$ devido seu tamanho reduzido, variando de 50 a $1000 \mathrm{~nm}$ e biocompatibilidade. ${ }^{6,7}$

Diferentes métodos de produção de NLS permitem incorporar princípios ativos hidrossolúveis ${ }^{8}$ e lipossolúveis. ${ }^{9}$ No entanto, devido à natureza lipídica da matriz, as NLS são particularmente adequadas para veicular ativos apolares, para os quais evidenciam uma capacidade de carga elevada. ${ }^{10}$ A Tabela 1 apresenta alguns exemplos de fármacos, recentemente incorporados em NLS, assim como a composição lipídica dessas partículas. A preparação das NLS pode ser realizada por fusão-emulsificação, por emulsificação-evaporação do solvente, ou a partir de microemulsões. ${ }^{2,11}$

\section{PREPARAÇÃO DAS NLS POR FUSÃO-EMULSIFICAÇÃO}

A preparação de NLS por fusão-emulsificação consiste na fusão prévia do lipídio, incorporando o princípio ativo por dissolução ou por dispersão. Para aumentar a estabilidade da emulsão, geralmente

*e-mail: eliana@ufp.edu.pt é adicionada fosfatidilcolina ao lipídio fundido. Em seguida, esta fase é emulsificada numa fase aquosa, que contém um tensoativo do tipo O/A como, por exemplo, um polissorbato ${ }^{16,23,26,27}$ ou um

Tabela 1. Fármacos incorporados em NLS e sua composição

\begin{tabular}{|c|c|c|}
\hline $\begin{array}{l}\text { Fármacos incorporados } \\
\text { em NLS }\end{array}$ & Composição & Ref. \\
\hline Vários corticosteroides & $\begin{array}{l}\text { Precirol }^{\circledR} \text { ATO 5; Compritol }{ }^{\circledR} 888 \text { ATO; } \\
\text { Dynasan }^{\circledR} 114, \text { Dynasan }^{\circledR} 118 \text { Softisan }^{\circledR} \\
\text { 142, Tegin }\end{array}$ & 12 \\
\hline Lopinavir & Compritol $^{\circledast} 888$ ATO & 13 \\
\hline Carvedilol & Ácido esteárico & 14 \\
\hline Luteína & Peceol $^{\circledR}$ & 15 \\
\hline Saquinavir & $\begin{array}{l}\text { Compritol }{ }^{\circledast} 888 \text { ATO e manteiga de } \\
\text { cacau }\end{array}$ & 16 \\
\hline Sinvastatina & Monoestearato de glicerina & 17 \\
\hline Gatifloxacina & Compritol $^{\circledR}$ ATO 888 & 18 \\
\hline 5 flurouracilo & Dynasan $^{\circledast} 114$ and 118 & 19 \\
\hline Diazepam & Compritol $^{\circledR}$ ATO 888; Imwitor ${ }^{\circledR}$ 900K & 20 \\
\hline Dexametasona & Compritol $^{\circledast} 888$ ATO & 21 \\
\hline Vinpocetina & Monoestearato de glicerina & 22 \\
\hline Cetoprofeno & Cera de carnaúba & 23 \\
\hline Itraconazol & Tristearina e triolina & 24 \\
\hline Budesonida & Compritol $^{\circledR} 888$ & 25 \\
\hline
\end{tabular}


polaxamer. ${ }^{15}$ No final, a emulsão recém-preparada é mantida à temperatura ambiente para a solidificação do lipídio e obtenção da dispersão aquosa de NLS. ${ }^{28}$ Para a emulsificação do lipídio fundido com a fase aquosa faz-se uso de ultrassons ${ }^{20,22,29}$ ou de agitação mecânica, ${ }^{30,31}$ podendo ainda proceder-se, adicionalmente, a uma operação complementar de homogeneização à alta pressão (HAP). ${ }^{32-34}$ Estes procedimentos provocam a ruptura das gotículas lipídicas de maiores dimensões, originando gotículas pequenas, dispersas no meio da fase aquosa. ${ }^{35}$

Os ultrassons e a agitação mecânica são métodos simples, mas apresentam a desvantagem de originarem NLS com dimensões heterogêneas. ${ }^{36}$ Além disso, o uso de ultrassons é sempre acompanhado por um risco de contaminação por titânio e pela possibilidade de degradação do princípio ativo, devido à elevada força de cavitação aplicada. Para se obter partículas lipídicas de dimensões nanométricas são requeridos períodos de agitação superiores a 15 min e a presença de fosfatidilcolina no lipídio fundido. No entanto, o aumento da concentração desta última provoca uma diminuição da cristalinidade das nanopartículas formadas. ${ }^{35}$ Além disso, as NLS que contêm uma concentração elevada de tensoativo não são apropriadas para administração intravenosa. ${ }^{37}$

A HAP constitui um método vantajoso para preparar NLS por fusão-emulsificação, uma vez que não apresenta dificuldades de transposição de escala e permite trabalhar em condições assépticas. ${ }^{38}$ Para se obter NLS com uma distribuição homogênea de tamanhos, toda a dispersão deverá ser sujeita à mesma intensidade de energia. Caso contrário, as gotículas localizadas em diferentes volumes da amostra estarão expostas a diferentes forças de dispersão, originando NLS com dimensões muito heterogêneas. A HAP caracteriza-se pela aplicação da mesma tensão de corte a toda a amostra, devido às dimensões reduzidas do orifício de saída do homogeneizador $(\leq 25-30 \mu \mathrm{m}) .^{38,39}$

A concentração lipídica das emulsões destinadas à preparação de NLS por fusão-emulsificação varia, em regra, entre 5 e $10 \%(\mathrm{~m} / \mathrm{m}),{ }^{26,40}$ podendo, no entanto, atingir valores compreendidos entre 30 e $50 \%{ }^{41}$

A HAP pode ser realizada a uma temperatura superior ou inferior à temperatura de fusão do lipídio. ${ }^{42-44} \mathrm{Na}$ primeira situação, denominase HAP realizada a quente e na segunda, HAP realizada a frio. $\mathrm{Na}$ HAP a quente, o lipídio é previamente fundido a uma temperatura de cerca de 5 a $10{ }^{\circ} \mathrm{C}$ acima do seu ponto de fusão, incorporando-se, em seguida, o princípio ativo. ${ }^{45}$

A preparação de NLS por HAP a quente ${ }^{38}$ é realizada fundindo o lipídio, contendo o princípio ativo, seguindo-se a sua dispersão por agitação mecânica ${ }^{46}$ ou por ultrassons, ${ }^{32,33,47}$ numa solução aquosa, aquecida à mesma temperatura, que contém um tensoativo do tipo O/A. Obtém-se, desta forma, uma "pré-emulsão", que, em seguida, é submetida à HAP, realizada a uma temperatura compreendida entre 70 e $90{ }^{\circ} \mathrm{C}$, resultando uma nanoemulsão do tipo O/A. No final, a nanoemulsão é resfriada a uma temperatura igual ou inferior à temperatura ambiente (entre 20 e $25^{\circ} \mathrm{C}$ ), ${ }^{26,48}$ seguindo-se a solidificação do lipídio e a obtenção da dispersão aquosa de NLS. A etapa de homogeneização pode ser repetida várias vezes. No entanto, deve ter-se em conta que a HAP, por si só, aumenta a temperatura da amostra em cerca de 10 a $20^{\circ} \mathrm{C}$ a cada ciclo de homogeneização. Em muitos casos, são suficientes 2 a 5 ciclos de homogeneização, a 500-1500 bar. ${ }^{35}$

A HAP pode ser realizada com o lipídio no estado sólido. A preparação de NLS por HAP a frio consiste em fundir previamente o lipídio, onde se incorpora rapidamente o princípio ativo, promovendo, em seguida, o resfriamento em gelo seco ou nitrogênio líquido. Uma velocidade de resfriamento elevada favorece a distribuição homogênea do princípio ativo no interior da matriz lipídica. ${ }^{49}$ Após solidificação, o material é fragmentado, recorrendo-se, por exemplo, a um almofariz ou moinho. ${ }^{50,51}$ Deste processo resultam micropar- tículas com dimensões entre 50 e $100 \mu \mathrm{m},{ }^{45}$ as quais se dispersam numa solução aquosa resfriada, contendo um tensoativo do tipo O/A, de modo a formar uma "pré-suspensão". Uma vez preparada, esta pré-suspensão é submetida à HAP à temperatura ambiente ou abaixo desta, obtendo-se uma dispersão aquosa de NLS. A realização da HAP a baixas temperaturas aumenta a fragilidade do lipídio, facilitando a obtenção de partículas lipídicas de menores dimensões. ${ }^{49}$

Em comparação com a HAP a quente, a HAP realizada a frio requer um maior número de ciclos de homogeneização (em regra 10 ciclos) e origina NLS de dimensões superiores e mais heterogêneas. ${ }^{45,49}$ Como a HAP aumenta a temperatura da amostra em cerca de 10 a $20^{\circ} \mathrm{C}$, em cada ciclo, a diferença entre o ponto de fusão do lipídio e a temperatura de homogeneização tem que ser suficientemente elevada, de modo a garantir a não ocorrência da fusão do lipídio durante o processo de HAP. ${ }^{52}$ As características físico-químicas das NLS obtidas por fusão-emulsificação são afetadas por um conjunto de parâmetros tecnológicos, que incluem a solubilidade do princípio ativo, o polimorfismo do lipídio, a natureza e concentração do lipídio e do tensoativo, a temperatura, a força de cisalhamento e o número de ciclos de homogeneização efetuados.

\section{Solubilidade do princípio ativo}

O princípio ativo pode ser incorporado no lipídio fundido, dissolvendo-se ou dispersando-se, independente de ser lipo- ou hidrossolúvel. Durante a preparação das NLS por HAP realizada a quente, o princípio ativo encontra-se dissolvido no lipídio fundido, numa concentração próxima da saturação, observando-se a sua difusão para a fase externa aquosa. Este fenômeno é tanto mais acentuado quanto maior for a hidrossolubilidade do princípio ativo, a temperatura da fase aquosa e a concentração do tensoativo nesta fase. ${ }^{35}$ Após a homogeneização cria-se uma região de sobressaturação do princípio ativo à volta das gotículas de lipídio fundido recém-formadas. Durante o resfriamento do sistema, a solubilidade do princípio ativo na fase externa aquosa diminui, sendo, em consequência, redistribuída na superfície das NLS, antes de o lipídio recristalizar completamente. Formam-se, assim, NLS constituídas por um núcleo lipídico revestido por uma parede contendo princípio ativo.

Devido à natureza aquosa da fase externa da "pré-emulsão", se a preparação das NLS for realizada por HAP a quente obtémse uma eficiência de encapsulação e carregamento mais elevadas para princípios ativos hidrófobicos. Estes princípios ativos têm menor tendência para abandonar o lipídio fundido, difundindo-se para a fase externa aquosa, contrariamente ao que sucede com os princípios ativos hidrofílicos. ${ }^{35}$ Para se aumentar a eficiência de encapsulação das substâncias hidrossolúveis, promove-se o aumento da sua solubilidade no lipídio fundido, por conjugação com pares iônicos, ${ }^{53}$ ou adicionando-se ao lipídio fundido um tensoativo, como a fosfatidilcolina, ou ainda recorrendo-se a lipídios com propriedades solubilizantes (como os mono e os diacilgliceróis) para formar a matriz lipídica sólida. A HAP realizada a frio proporciona um aumento da incorporação dos princípios ativos hidrofílicos nas NLS. ${ }^{49}$ Durante a homogeneização, o lipídio encontra-se no estado sólido, pelo que é minimizada a difusão do princípio ativo da matriz lipídica sólida para a fase externa aquosa, contrariamente ao que sucede com a HAP realizada a quente. A mobilidade das moléculas dos princípios ativos é menor na matriz lipídica sólida do que no lipídio fundido, pelo que, durante a HAP realizada a frio, menor será a quantidade de ativos que se perde para a fase aquosa. Além disso, esta fase pode ser substituída por outros líquidos, como os polietilenoglicóis de massa molar baixa, nos quais o princípio ativo evidencia menor difusão e solubilidade, o que contribui para a sua retenção na matriz lipídica sólida. ${ }^{45}$ 


\section{Polimorfismo do lipídio}

O processo de preparação de NLS inicia-se com a fusão do lipídio, seguida do resfriamento para originar NLS. Ao iniciar o processo de solidificação, a viscosidade do lipídio fundido aumenta progressivamente, tornando-se mais difícil acomodar as moléculas lipídicas, o que origina, no final, cristais lipídicos com diferentes graus de organização tridimensional, isto é, com diferentes formas polimórficas. ${ }^{54}$ Este fenômeno é habitualmente conhecido por recristalização do lipídio. O grau de cristalinidade das NLS recém-formadas depende da organização tridimensional das cadeias hidrocarbonadas das moléculas na matriz lipídica solidificada. ${ }^{55}$

Os lipídios podem apresentar polimorfismo, isto é, se cristalizar em formas distintas, criando estruturas diferenciadas, alterando a liberação do princípio ativo encapsulado. Essas formas polimórficas podem ser identificadas por padrões de difração de raios $\mathrm{X}$, onde os espaçamentos longos fornecem informações sobre a distância entre os cristais planos (comprimento de cadeia), enquanto os espaçamentos curtos informam sobre a estrutura da sub-célula (distância entre cadeias). ${ }^{56}$ Os lipídios podem cristalizar em estruturas tridimensionais do tipo $\alpha, \beta^{\prime}, \beta$. A forma $\alpha$ (hexagonal) é a menos estável, com menor ponto de fusão e calor latente. A forma $\beta$ (triciclíca) é a mais estável, com maior ponto de fusão e maior calor latente. As transformações de $\alpha$ para $\beta^{\prime}$ (ortorrômbica) e desta para $\beta$ ocorrem nesta ordem e são irreversíveis. ${ }^{57,58}$ Desta forma, para uma aplicação industrial das partículas lipídicas sólidas é necessário controlar este polimorfismo. A estrutura polimórfica tem influência direta na eficiência de encapsulação e na expulsão do ativo durante o processo de estocagem, sendo muito importante a sua caracterização. ${ }^{54,59}$ Os espaços dessas moléculas estão descritos na Tabela 2.

Tabela 2. Estrutura tridimensional das três formas mais comuns de polimorfismo em triacilgliceróis

\begin{tabular}{|c|c|c|c|}
\hline Modificações & $\alpha$ & $\beta^{\prime}$ & $\beta$ \\
\hline cristal & Hexagonal $(\mathrm{H})$ & $\begin{array}{c}\text { Ortorrômbica } \\
\text { perpendicular }(\mathrm{O} \perp)\end{array}$ & $\begin{array}{l}\text { Triclínica paralela } \\
\qquad(\mathrm{T} / /)\end{array}$ \\
\hline subcélulas & $\begin{array}{l}\text { Ortorrômbica } \\
0,415-0,42 \mathrm{~nm}\end{array}$ & $\begin{array}{l}\text { Ortorrômbica } \\
0,42-0,43 \mathrm{~nm} \\
0,37-0,40 \mathrm{~nm}\end{array}$ & $\begin{array}{l}\text { Triclínica } \\
0,46 \mathrm{~nm}\end{array}$ \\
\hline
\end{tabular}

A organização tridimensional que a matriz lipídica adquire durante a solidificação depende da velocidade de resfriamento do lipídio e da sua composição, e constitui um fator muito importante para a incorporação do princípio ativo nas NLS. Quando a velocidade de resfriamento é lenta, as cadeias hidrocarbonadas dos lipídios podem se rearranjar numa forma mais ordenada e estável. Pelo contrário, quando a velocidade de resfriamento é elevada, a solidificação da matriz ocorre também rapidamente, rearranjando-se numa forma polimórfica mais instável. Assim, na preparação de NLS por HAP realizada a quente, como a velocidade de resfriamento é elevada, as partículas recém-formadas adquirem, preferencialmente, a forma polimórfica $\beta .{ }^{60}$ No que se refere à composição lipídica, as matrizes com um conteúdo elevado de diacilgliceróis (> 50\%) cristalizam na forma polimórfica metaestável $\beta,{ }^{6}{ }^{6}$ que se caracteriza por alguma imperfeição na sua estrutura tridimensional. As matrizes lipídicas formadas por triacilgliceróis, esterificados com um único tipo de ácido graxo, cristalizam, normalmente, no polimorfo mais estável $\beta$, que se caracteriza por um grau de organização elevado. Adicionalmente, são encontradas outras formas polimórficas em misturas de acilgliceróis que são habitualmente referidas como formas $\beta_{\mathrm{i}}{ }^{55}$

A estabilidade termodinâmica das NLS e o seu grau de empacotamento lipídico aumentam, enquanto a incorporação do princípio ativo diminui na seguinte ordem: forma polimórfica $\alpha$, forma poli- mórfica $\beta$, forma polimórfica $\beta$. Em regra, as moléculas lipídicas apresentam maior mobilidade quando se encontram em configurações termodinamicamente mais instáveis como, por exemplo, a forma polimórfica $\alpha$. Por esta razão, estas configurações apresentam um grau de empacotamento lipídico menor, sendo maior a sua capacidade para incorporar os princípios ativos. ${ }^{60}$ Portanto, para a preparação de NLS com capacidade de carga elevada, em particular quando se pretende incorporar ativos hidrofílicos, o lipídio deverá cristalizar, preferencialmente, na forma polimórfica mais instável, isto é, na forma $\alpha$, que deverá se manter durante o armazenamento. ${ }^{62,63}$

As transformações polimórficas podem ocorrer durante o armazenamento promovendo a instabilidade da dispersão coloidal, uma vez que estão associadas ao aumento do tamanho das partículas lipídicas. Durante o armazenamento, a ocorrência de transformações polimórficas é acompanhada por alterações na forma das partículas, designadamente das formas esféricas $(\alpha)$ para as formas achatadas (ß). ${ }^{64,65}$

O problema associado às modificações do lipídio não é sempre resolvido com a manipulação das formas $\alpha, \beta$ e $\beta$ '. A complexidade do sistema aumenta devido às subespécies polimórficas e às interações do lipídio com os agentes tensoativos. Para preparar NLS com capacidade de carga elevada utilizando formas polimórficas instáveis, é necessário o desenvolvimento de estratégias que previnam a transformação das formas instáveis para as formas mais estáveis, durante o armazenamento. ${ }^{66}$

\section{Natureza e concentração do lipídio}

A maioria dos lipídios utilizados na preparação de NLS consiste em misturas de vários compostos químicos. A natureza química dos lipídios selecionados e a sua concentração influenciam a incorporação dos princípios ativos e tamanho das NLS.

Os lipídios que dão origem a partículas altamente cristalinas, como os triacilgliceróis constituídos por um tipo de ácido gordo, originam estruturas organizadas, com poucos locais para acomodar as moléculas de princípios ativos, induzindo a sua expulsão da matriz lipídica sólida. ${ }^{67}$ Os lipídios de composição mais complexa, como as misturas de mono, di e triacilgliceróis e as misturas de diferentes ácidos graxos formam cristais com muitas imperfeições, oferecendo mais espaço para acomodar as moléculas de princípio ativo. ${ }^{68}$ Por esta razão, as misturas de acilgliceróis proporcionam uma capacidade de carga maior do que os lipídios puros.

Para se obter NLS com dimensões reduzidas, recorre-se a misturas de mono e diacilgliceróis, os quais apresentam propriedades tensoativas (EHL 2-5). ${ }^{20}$ Ahlin et al..$^{69}$ observaram que o tamanho médio das NLS de Witepsol ${ }^{\circledR}$ W35 (constituído por ácidos graxos de cadeia curta e quantidades elevadas de mono e diacilgliceróis) era significativamente menor $(117,0 \pm 1,8 \mathrm{~nm})$ do que o das NLS de Dynasan ${ }^{\circledR} 118$ (constituído por triestearina) $(175,1 \pm 3,5 \mathrm{~nm})$. Utilizando a HAP a quente, Siekmann e Westesen ${ }^{70}$ demonstraram que o tamanho médio das NLS aumenta com o aumento da temperatura de fusão dos lipídios usados para a preparação, devido ao aumento da viscosidade da fase interna da emulsão.

O aumento da concentração lipídica aumenta as dimensões das NLS, devido ao aumento da viscosidade da "pré-emulsão", tornando mais difícil a sua emulsificação na fase aquosa. ${ }^{71}$ Portanto, são formadas NLS de dimensões maiores. Como as emulsões contêm cerca de 5 a $10 \%(\mathrm{~m} / \mathrm{m})$ de lipídio, a sua viscosidade é suficientemente reduzida para serem homogeneizadas por HAP, obtendo-se NLS com dimensões reduzidas e com uma distribuição homogênea de tamanhos. Acima de $10 \%(\mathrm{~m} / \mathrm{m})$ de lipídio resultam NLS de dimensões mais elevadas e uma distribuição mais heterogênea de tamanhos. $^{52}$ 


\section{Natureza e concentração do tensoativo}

A escolha do tensoativo e da sua concentração são fatores que influenciam diretamente as características das NLS, designadamente as suas dimensões, a retenção do princípio ativo e a eficiência de encapsulação. ${ }^{65} \mathrm{Na}$ preparação de NLS por HAP a quente é frequente incorporar fosfolipídeos (como a fosfatidilcolina) ${ }^{72}$ no lipídio fundido e um estabilizante estereoquímico (por exemplo, um polaxamer) na fase aquosa externa. ${ }^{73}$ Deste modo, forma uma barreira mecânica e/ou elétrica que impede a coalescência das partículas durante a sua formação, e também a sua agregação durante o armazenamento. A carga elétrica da superfície das partículas provoca a repulsão, mantendo o sistema num estado termodinâmico mais estável. Se a fase interna da nanoemulsão O/A for suficientemente estável, as NLS obtidas após a recristalização do lipídio apresentarão menores dimensões e maior estabilidade durante o armazenamento, em comparação com formulações que contêm apenas um tensoativo incluído na fase aquosa. ${ }^{74,75}$

Se o aumento da concentração de fosfatidilcolina for acompanhado pelo aumento do tamanho das NLS, este se deve, provavelmente, à formação de múltiplas camadas de fosfatidilcolina à sua superfície ou à formação de lipossomas na fase aquosa. É de notar que as moléculas de fosfolipídio em excesso na fase interna se deslocam para a superfície das partículas lipídicas e/ou para a fase aquosa, organizando-se em bicamadas. As concentrações elevadas de tensoativo reduzem a tensão interfacial, aumentam a área de superfície e, em consequência, originam NLS de menores dimensões. ${ }^{76}$ No entanto, quando a fase aquosa externa contém moléculas de tensoativo em excesso podem se formar outras espécies coloidais (como, por exemplo, lipossomas), que podem incorporar moléculas de princípios ativos e diminuir a sua eficiência de encapsulação nas NLS. ${ }^{26}$

\section{Temperatura}

Na preparação de NLS por HAP realizada a quente, as temperaturas elevadas conduzem a uma diminuição do tamanho das partículas, uma vez que promovem uma diminuição da viscosidade da fase interna, originando também uma distribuição mais homogênea de tamanhos. ${ }^{77}$ Para iniciar a recristalização do lipídio, é necessário resfriar a nanoemulsão O/A a uma temperatura que depende da natureza do lipídio. Quando se utilizam acilgliceróis formados por ácidos graxos de cadeia curta, e com baixo ponto de fusão (próximo da temperatura ambiente), a recristalização deve ocorrer a um valor inferior a esta. Em alternativa, a recristalização do lipídio pode ser iniciada submetendo a nanoemulsão à liofilização.

\section{Tensão de cisalhamanto e número de ciclos de homogeneização}

Na preparação de NLS por HAP realizada a quente, para se reduzir as dimensões das gotículas de fase interna da "pré-emulsão" pode aumentar-se a tensão aplicada ou o número de ciclos de homogeneização. ${ }^{77}$ No entanto, o excesso de ciclos, o aumento da tensão aplicada ou do número de ciclos origina NLS de dimensões mais elevadas, devido à coalescência das gotículas lipídicas, que ocorre em virtude do aumento da energia cinética. ${ }^{38}$

Na preparação de NLS por HAP realizada a frio, o lipídio encontra-se no estado sólido, pelo que se torna necessária uma tensão superficial mais elevada e um maior número de ciclos de homogeneização para se obter partículas com dimensões nanométricas. ${ }^{49}$

\section{Problemas inerentes à preparação de NLS por fusão- emulsificação}

Os problemas inerentes à preparação de NLS por fusão-emul- sificação estão relacionados com o risco potencial de degradação do lipídio e/ou do princípio ativo e com a possível ocorrência do fenômeno de recristalização do lipídio.

\section{Degradação do lipídio elou do princípio ativo}

O risco de ocorrência de degradação do lipídio e/ou do princípio ativo durante a HAP está associado às elevadas temperaturas e tensões de cisalhamento aplicadas durante o processo. No caso de princípios ativos termossensíveis, a HAP realizada a frio tem vantagens, uma vez que o impacto térmico é apenas aplicado no início do processo, para promover a fusão do lipídio. ${ }^{49}$ No entanto, a HAP, por si só, aumenta a temperatura da amostra, pelo que, quando se processa a frio, deve garantir-se que a temperatura é suficientemente reduzida para evitar a fusão do lipídio. ${ }^{51}$

A HAP pode também ser responsável pela redução do massa molar do lipídio e do princípio ativo, já que uma tensão de cisalhamento elevada parece ser a principal causa para a formação de radicais livres, responsáveis pela peroxidação lipídica. ${ }^{45,49}$ A massa molar e a estrutura molecular do princípio ativo são os parâmetros mais importantes para avaliar a sua possível degradação pela aplicação de tensões de cisalhamento elevadas. Os princípios ativos de massa molar elevada e com cadeias longas são as mais sensíveis. Com efeito, a HAP parece causar a degradação do DNA e da albumina. No entanto, para a maior parte dos princípios ativos incorporados em NLS, a HAP não apresenta qualquer problema.

\section{Recristalização do lipídio}

Para preparar NLS por HAP a quente, a nanoemulsão O/A previamente obtida deverá ser resfriada, de modo a recristalizar o lipídio e, em consequência, originar a formação de partículas lipídicas solidificadas. Devido à quantidade elevada de moléculas de tensoativo, necessária para estabilizar a dispersão coloidal, e à complexidade química da mistura lipídica, a cristalinidade do lipídio sob a forma de NLS pode diferir consideravelmente do lipídio como matéria-prima. Devido à mistura complexa de lipídios, a diferença de temperatura entre os pontos de fusão e de cristalização da matriz lipídica (intervalo de resfriamento) pode atingir entre 30 e $40{ }^{\circ} \mathrm{C}$ nas dispersões lipídicas. Por exemplo, a temperatura de fusão da trilaurina é superior a $40{ }^{\circ} \mathrm{C}$. No entanto, nas NLS formadas por um sistema de tensoativos constituído por um fosfolipídio e tiloxapol, o lipídio recristaliza a temperaturas inferiores ao ponto de congelação da água. ${ }^{65} \mathrm{~A}$ estabilidade das dispersões coloidais de NLS está, portanto, condicionada pela ocorrência de fusões super-resfriadas e pelas transformações polimórficas que o lipídio sofre, quando da sua recristalização sob a forma de partículas sólidas e durante o armazenamento.

\section{Fusões super-resfriadas}

Na sequência de uma HAP realizada a quente podem resultar fusões super-resfriadas, em vez de dispersões aquosas de NLS. As fusões super-resfriadas são sistemas heterogêneos semelhantes a emulsões do tipo O/A, uma vez que o lipídio se mantém no estado líquido e disperso na fase aquosa, embora a preparação seja armazenada a uma temperatura inferior ao ponto de fusão do lipídio. Estes sistemas resultam da recristalização do lipídio ser consideravelmente retardada, após a realização da HAP a quente, devido ao baixo ponto de fusão e à composição complexa da mistura lipídica utilizada. Se a recristalização não for induzida artificialmente, as dispersões lipídicas podem permanecer super-resfriadas durante vários meses. A ocorrência de fusões super-resfriadas aumenta com a diminuição das dimensões das gotículas de lipídio fundido emulsificadas na fase aquosa. Para o lipídio iniciar a recristalização é necessária a existência de um determinado número de núcleos de cristalização presentes na emulsão. A formação desses núcleos é menor com a diminuição 
das dimensões das gotículas e, como consequência, é retardado o processo de recristalização.

Os aumentos da temperatura e da tensão de cisalhamento aplicada podem originar fusões super-resfriadas, devido à formação de gotículas lipídicas de dimensões reduzidas. Pode-se verificar, também, um atraso na recristalização com o aumento da concentração de tensoativo e com a diminuição da concentração do lipídio na formulação.

Em comparação com as formas polimórficas que o lipídio pode adquirir $(\alpha, \beta$ ' ou $\beta)$, a estabilidade termodinâmica e o grau de empacotamento lipídico são mais baixos nas fusões super-resfriadas. ${ }^{54}$ Por esta razão, estas últimas não conseguem imobilizar as moléculas de princípio ativo tão fortemente quanto as partículas sólidas, não podendo, por isso, ser utilizadas para modificar a cedência in vivo das moléculas incorporadas. Além disso, mesmo que fosse possível recristalizar o lipídio e formar NLS a partir das fusões super-resfriadas, após a administração in vivo ocorreria a sua fusão, uma vez que a temperatura corporal é superior ao ponto de fusão do lipídio.

\section{PREPARAÇÃO POR EMULSIFICAÇÃO-EVAPORAÇÃO DO SOLVENTE}

A preparação de NLS por emulsificação-evaporação do solvente consiste na preparação de uma fase orgânica, constituída pelo lipídio dissolvido num solvente orgânico imiscível com a água como, por exemplo, a acetona, o diclorometano ${ }^{78}$ ou clorofórmio. ${ }^{79}$ Nesta fase é incorporado o princípio ativo, por dissolução ou por dispersão, e adicionada a fosfatidilcolina para aumentar a estabilidade termodinâmica da emulsão preparada. A fase orgânica é depois emulsificada numa fase aquosa, que contém um tensoativo do tipo O/A como, por exemplo, o polaxamer $188,{ }^{15}$ obtendo-se uma emulsão do tipo O/A.

Para se proceder à evaporação do solvente orgânico, a emulsão obtida é diluída num volume elevado de fase aquosa e submetida à agitação a uma temperatura compreendida entre 0 e $2{ }^{\circ} \mathrm{C}$, podendo decorrer à pressão reduzida. As NLS formam-se em consequência da precipitação do lipídio na fase externa aquosa. A evaporação do solvente orgânico deve se realizar o mais rapidamente possível, de modo a evitar a agregação das NLS recém-formadas. Os princípios ativos hidrossolúveis, em vez de serem dispersos na fase orgânica podem, como alternativa, ser dissolvidos numa fase aquosa, que, posteriormente, vai constituir a fase interna de uma emulsão múltipla $\mathrm{A} / \mathrm{O} / \mathrm{A} .{ }^{80}$ Este processo é particularmente adequado para a incorporação de peptídeos e proteínas em NLS. Neste caso, como a fase aquosa interna tem tendência para coalescer durante a agitação, a presença de um tensoativo na fase orgânica é essencial para aumentar a estabilidade da emulsão primária $\mathrm{A} / \mathrm{O}$ e, em consequência, da emulsão múltipla A/O/A. ${ }^{81}$

Em comparação com o processo de preparação das NLS por fusão-emulsificação, a emulsificação-evaporação do solvente origina partículas com dimensões mais reduzidas, devido à menor viscosidade da fase interna, que resulta do lipídio se encontrar dissolvido num solvente orgânico em lugar de fundido. Este método apresenta a vantagem de evitar a exposição dos princípios ativos a temperaturas elevadas, apresentando, no entanto, o inconveniente relacionado com o uso de solventes orgânicos. ${ }^{8}$

As características físico-químicas das NLS obtidas por emulsificação-evaporação do solvente são afetadas por um conjunto de parâmetros tecnológicos, que incluem a solubilidade do princípio ativo, o polimorfismo do lipídio e, ainda, a natureza e concentração do lipídio e do agente tensoativo.

\section{Solubilidade do princípio ativo}

Os princípios ativos hidrofóbicos dissolvem-se na fase interna orgânica da emulsão e não sofrem difusão para a fase externa aquosa, obtendo-se, por isso, uma incorporação elevada. No caso dos princípios ativos hidrofílicos, estas evidenciam uma tendência elevada para abandonar a fase interna orgânica, pelo que a sua eficiência de encapsulação é significativamente reduzida. Para ultrapassar este problema, estas substâncias, em particular, os peptídeos e as proteínas, podem ser dissolvidas numa fase aquosa interna, formando-se uma emulsão múltipla A/O/A, com a qual se preparam as NLS. Em comparação com o método de fusão-emulsificação anteriormente descrito, a emulsificação-evaporação do solvente origina NLS com eficiência de encapsulação menores para os princípios ativos hidrossolúveis. Neste caso, a fase interna é menos viscosa, uma vez que o lipídio se encontra dissolvido num solvente orgânico, enquanto no método de fusão-emulsificação, o lipídio está fundido, contribuindo para reduzir a difusão do princípio ativo para a fase externa aquosa.

\section{Polimorfismo do lipídio}

Devido à difusão lenta do solvente orgânico através da fase aquosa, a solidificação das NLS obtidas por emulsificação-evaporação do solvente é mais lenta, em comparação com as preparadas por fusãoemulsificação. Em consequência, as moléculas lipídicas podem se rearranjar numa forma termodinâmica mais estável, adquirindo a forma polimórfica $\beta$, à qual está associada uma menor capacidade para incorporar os princípios ativos.

\section{Natureza e concentração do lipídio}

A natureza do lipídio influencia também as características físicoquímicas das NLS preparadas por emulsificação-evaporação do solvente. Com efeito, os lipídios de composição complexa, como as misturas de mono, di e triacilgliceróis, e os que contêm ácidos graxos com cadeia de diferentes comprimentos, originam NLS com muitas imperfeições, oferecendo mais espaço para acomodar as moléculas do princípio ativo. O tamanho médio das NLS aumenta com o aumento da concentração lipídica na emulsão, devido ao aumento da viscosidade da fase interna. Em regra, são utilizadas concentrações compreendidas entre 0,5 e $2,5 \%(\mathrm{~m} / \mathrm{v})$. Para se obter NLS de dimensões menores são utilizadas concentrações lipídicas inferiores a $5 \%(\mathrm{~m} / \mathrm{v}){ }^{80}$

\section{Natureza e concentração do tensoativo}

O tamanho médio das NLS e a extensão de incorporação dos princípios ativos por emulsificação-evaporação do solvente depende do tipo de agentes tensoativos utilizados. A presença de um agente tensoativo do tipo O/A na fase externa aquosa é fundamental para a formação da emulsão O/A. O aumento da sua concentração provoca uma diminuição da tensão interfacial entre ambas as fases, contribuindo para diminuir as dimensões das NLS.

Verifica-se, igualmente, uma redução das dimensões das NLS, quando se usa um sistema de tensoativos, em que um está incluído na fase interna, como um fosfolipídio, e outro, na fase externa, como um sal biliar. ${ }^{76,82}$ Quando o princípio ativo se encontra disperso, em lugar de dissolvido, na fase orgânica, a sua tendência para se difundir para a fase aquosa é maior, reduzindo a sua incorporação nas NLS. A utilização de um agente tensoativo na fase interna aumenta a molhabilidade das partículas sólidas do princípio ativo, contribuindo para a sua permanência nesta fase e permitindo aumentar a eficiência de encapsulação.

\section{PREPARAÇÃO A PARTIR DE MICROEMULSÕES}

As microemulsões são sistemas heterogêneos termodinamicamente estáveis, que podem ser formados espontaneamente devido 
a uma tensão interfacial reduzida entre uma fase interna e uma fase externa. Para se preparar a microemulsão, o lipídio é inicialmente fundido, incorporando-se depois o princípio ativo por dissolução ou por dispersão, consoante esta é lipo ou hidrossolúvel. Simultaneamente, prepara-se, à mesma temperatura, uma fase aquosa, contendo um agente tensoativo do tipo O/A. Em seguida, submete-se à emulsificação do lipídio fundido na fase aquosa, sob agitação mecânica intensa, obtendo-se uma microemulsão O/A termodinamicamente estável. ${ }^{83}$

A microemulsão $\mathrm{O} / \mathrm{A}$, em regra aquecida a uma temperatura compreendida entre 60 e $70^{\circ} \mathrm{C},{ }^{84-86}$ é diluída sob agitação mecânica, num volume elevado de água ${ }^{87}$ ou de uma solução aquosa resfriada a uma temperatura compreendida entre 2 e $3{ }^{\circ} \mathrm{C}$. A razão entre os volumes da microemulsão e da solução aquosa está compreendida entre 1:2 e 1:100 (v/v). Desta forma, assegura-se que as dimensões reduzidas das NLS são devidas à precipitação do lipídio na solução aquosa resfriada e não induzidas mecanicamente pelo processo de agitação. Devido à etapa de diluição, a concentração lipídica da microemulsão é consideravelmente mais baixa, em comparação com a das emulsões obtidas no método de fusão-emulsificação. ${ }^{83} \mathrm{As}$ características físico-químicas das NLS obtidas a partir de microemulsões são afetadas, não somente pelos fatores que interferem na preparação da microemulsão, mas também pelas condições experimentais em que decorre a sua diluição na solução aquosa, designadamente pela temperatura desta última e pelo seu volume e, ainda, pelo modo como se processa a diluição. Os principais parâmetros que afetam a eficiência de encapsulação são o carregamento e o tamanho das NLS.

\section{TEMPERATURA E VOLUME DA SOLUÇÃO AQUOSA}

As NLS são formadas devido ao contato rápido entre a microemulsão aquecida e a solução aquosa resfriada, pelo que a diferença de temperatura entre ambas e o volume da solução aquosa são parâmetros que afetam as dimensões das partículas. A diferença de temperatura entre a microemulsão recém-preparada e a solução aquosa resfriada é o principal parâmetro que afeta as dimensões das NLS. Uma diferença elevada facilita a recristalização rápida do lipídio e previne a agregação das NLS recém-formadas. Por esta razão, são obtidas NLS com menores dimensões quando é maior a diferença de temperatura entre a microemulsão aquecida e a solução aquosa resfriada ${ }^{83} \mathrm{Um}$ volume mais elevado de solução aquosa resfriada diminui o risco de agregação das NLS recém-formadas, enquanto um menor volume poderá não ser suficiente para dispersar a totalidade da microemulsão, obtendo-se NLS de maiores dimensões. ${ }^{87}$ A diluição da microemulsão $\mathrm{O} / \mathrm{A}$ numa solução aquosa resfriada processa-se sob agitação mecânica. Embora a agitação não seja o fator responsável pelas dimensões reduzidas das NLS obtidas, este processo pode não ser adequado para o controle das dimensões das partículas devido à agregação que podem sofrer, originando uma distribuição mais heterogênea de tamanhos. A utilização de dispositivos capilares constitui uma alternativa vantajosa para o controle das dimensões das NLS, já que proporciona uma diluição gradual e controlada da microemulsão.

Marengo et al ${ }^{88}$ desenvolveram um aparelho para a preparação industrial de NLS, que permite a diluição de um volume superior a $100 \mathrm{~mL}$ de microemulsão líquida aquecida numa solução aquosa resfriada. A microemulsão é colocada numa câmara de alumínio, revestida por uma parede termostatizada, e esterilizada por filtração através uma membrana filtrante com $0,22 \mu \mathrm{m}$ de abertura do poro. Posteriormente, a microemulsão é conduzida através de uma agulha, diretamente para a solução aquosa resfriada, sob agitação magnética a 300 rpm. O diâmetro médio e a distribuição de tamanhos das NLS estão condicionados pelo diâmetro da agulha utilizada, pela temperatura e pelo volume da solução aquosa. ${ }^{88}$ Para um determinado valor de temperatura e volume de solução, o aumento do diâmetro da agulha origina NLS maiores, uma vez que as gotículas emulsificadas são também maiores.

\section{CONCLUSÕES}

As NLS podem ser usadas para as mesmas finalidades das nanopartículas poliméricas. Por esta razão, têm sido igualmente objeto de estudo, considerando a sua administração por vias não parenterais (sob a forma de comprimidos, pellets, cápsulas, hidrogéis e cremes) e por vias parenterais. Após a administração oral, pode-se conseguir uma liberação modificada dos princípios ativos para a mucosa gastrointestinal e reduzir a variabilidade da absorção, devido à natureza lipídica da sua matriz. A absorção pode ser melhorada, em comparação com as nanopartículas poliméricas, devido às propriedades adesivas da matriz lipídica à mucosa gastrointestinal. Além disso, os lipídios das NLS podem ser processados e absorvidos, tal como os lipídios provenientes da alimentação. Assim, os princípios ativos podem ser absorvidos juntamente com os lipídios, com aumento da sua biodisponibilidade. A maior vantagem das NLS para administração tópica reside no fato de protegerem dos princípios ativos contra a degradação química e modularem a sua liberação para camadas particulares da pele. Em muitos casos, para se conseguir uma formulação para a administração tópica é necessária a incorporação das dispersões aquosas de NLS em hidrogéis ou em cremes.

No que se refere à administração parenteral, as NLS têm sindo estudadas para o direcionamento de princípios ativos para os pulmões, baço e cérebro. As propriedades físico-químicas das NLS dependem do método de preparação utilizado, o qual, por sua vez, é influenciado por vários parâmetros tecnológicos. Como a matriz destes sistemas é de natureza lipídica, os métodos de preparação ocorrem em meio orgânico e evidenciam melhores resultados para princípios ativos de natureza hidrofóbica. Devido ao fenômeno de recristalização do lipídio, necessário para a solidificação da matriz e formação das nanopartículas, as propriedades físico-químicas das NLS estão condicionadas pela ocorrência de transformações polimórficas, que dependem do tipo de lipídio utilizado. O tipo de forma polimórfica que o lipídio adquire condiciona a capacidade de carga das NLS. Os lipídios que cristalizam em formas altamente organizadas, como os triacilgliceróis, constituídos por um único tipo de ácido graxo, originam sistemas com poucos locais para acomodar os princípios ativos, induzindo a sua expulsão da matriz lipídica sólida. Os lipídios de composição mais complexa, como as misturas de mono, di e triacilgliceróis, formam estruturas com muitas imperfeições, originando NLS com maior capacidade para incorporar os princípios ativos.

As NLS podem ser preparadas a partir de métodos que se baseiam na fusão-emulsificação, sendo particularmente importantes aqueles que recorrem à HAP realizada a quente ou a frio. Os parâmetros que concorrem para a otimização das formulações incluem, para além da natureza e concentração do lipídio e do tensoativo, a temperatura, a tensão de superficial e o número de ciclos de homogeneização. Este método apresenta o inconveniente associado ao risco de degradação do lipídio e/ou do princípio ativo, devido às elevadas temperaturas e tensões superficiais aplicadas durante o processo, bem como ao risco de ocorrência de transformações polimórficas durante o armazenamento das NLS.

A preparação por emulsificação-evaporação do solvente é realizada no meio de emulsões do tipo O/A ou A/O/A, sendo particularmente adequado para incorporar substâncias termossensíveis, uma vez que durante o processo de preparação não se aplicam temperaturas elevadas. Este método apresenta o inconveniente relacionado com a utilização de solventes orgânicos, nos quais deve ser solubilizado o material lipídico.

Finalmente, a preparação de NLS a partir de microemulsões requer a utilização de um dispositivo apropriado para dispersar a 
microemulsão aquecida numa solução aquosa resfriada, sendo, por isso, um método influenciado pela temperatura e pelo volume da solução aquosa e pelas dimensões do dispositivo capilar utilizado. Neste método, as dimensões reduzidas das NLS são devidas à precipitação do lipídio na solução aquosa resfriada e não induzidas mecanicamente pelo processo de agitação.

\section{AGRADECIMENTOS}

À Fundação para a Ciência e a Tecnologia (FCT) do Ministério da Ciência e Tecnologia (PTDC/SAU-FAR/113100/2009), à Fundação de Amparo à Pesquisa do Estado de São Paulo (FAPESP/Brasil) e à Coordenação de Aperfeiçoamento de Pessoal de Nível Superior (CAPES, Brasil).

\section{REFERÊNCIAS}

1. Trevaskis, N. L.; Charman, W. N.; Porter, C. J.; Adv. Drug Deliv. Rev. 2008, 60, 702 .

2. Souto, E. B.; Tese de Mestrado, Faculdade de Farmácia do Porto, Portugal, 2003.

3. Rawat, M. K.; Jain, A.; Singh, S.; J. Pharm. Sci. 2011, 100, 2406.

4. Nayak, A. P.; Tiyaboonchai, W.; Patankar, S.; Madhusudhan, B.; Souto, E. B.; Colloids Surf., B 2010, 81, 263.

5. Zhang, J.; Smith, E.; J. Pharm. Sci. 2011, 100, 896.

6. Araujo, J.; Gonzalez-Mira, E.; Egea, M. A.; Garcia, M. L.; Souto, E. B.; Int. J. Pharm. 2010, 393, 167.

7. Muchow, M.; Maincent, P.; Muller, R. H.; Drug Dev. Ind. Pharm. 2008, 34, 1394.

8. Singh, S.; Dobhal, A. K.; Jain, A.; Pandit, J. K.; Chakraborty, S.; Chem. Pharm. Bull. (Tokyo) 2010, 58, 650.

9. Bunjes, H.; J. Pharm. Pharmacol. 2010, 62, 1637.

10. Wang, T.; Wang, N.; Zhang, Y.; Shen, W.; Gao, X.; Li, T.; Colloids Surf., B 2010, 79, 254.

11. Souto, E. B.; Tese de Doutorado, Faculdade de Farmácia do Porto, Portugal, 2003.

12. Jensen, L. B.; Magnussson, E.; Gunnarsson, L.; Vermehren, C.; Nielsen, H. M.; Int. J. Pharm. 2009, 390, 53.

13. Aji Alex, M. R.; Chacko, A. J.; Jose, S.; Souto, E. B.; Eur. J. Pharm. Sci. 2011, 18, 11.

14. Chakraborty, S.; Shukla, D.; Vuddanda, P. R.; Mishra, B.; Singh, S.; Colloids Surf., B 2010, 81, 563.

15. Yoo, J. H.; Shanmugam, S.; Thapa, P.; Lee, E. S.; Balakrishnan, P.; Baskaran, R.; Yoon, S. K.; Choi, H. G.; Yong, C. S.; Yoo, B. K.; Han, K.; Arch. Pharm. Res. 2010, 33, 417.

16. Kuo, Y. C.; Chen, H. H.; Int. J. Pharm. 2009, 365, 206.

17. Shah, M.; Pathak, K.; AAPS Pharm. Sci. Tech. 2010, 11, 489.

18. Kalam, M. A.; Sultana, Y.; Ali, A.; Aqil, M.; Mishra, A. K.; Chuttani, K.; J. Drug Targeting 2010, 18, 191.

19. Yassin, A. E.; Anwer, M. K.; Mowafy, H. A.; El-Bagory, I. M.; Bayomi, M. A.; Alsarra, I. A.; Int. J. Med. Sci. 2010, 7, 398.

20. Abdelbary, G.; Fahmy, R. H.; AAPS Pharm. Sci. Tech. 2009, 10, 211.

21. Chen, G.; Hou, S. X.; Hu, P.; Hu, Q. H.; Guo, D. D.; Xiao, Y.; Nan Fang Yi Ke Da Хие Хие Bao. 2008, 28, 1022.

22. Luo, Y.; Chen. D.; Ren, L.; Zhao, X.; Qin, J.; J. Controlled Release 2006, 114, 53.

23. Kheradmandnia, S.; Vasheghani-Farahani, E.; Nosrati, M.; Atyabi, F.; Nanomedicine 2010, 6, 753.

24. Kim, J. K.; Park, J. S.; Kim, C. K.; Int. J. Pharm. 2010, 383, 209.

25. Mezzena, M.; Scalia, S.; Young, P. M.; Traini, D.; AAPS J. 2009, 11, 771.

26. Helgason, T.; Awad, T. S.; Kristbergsson, K.; McClements, D. J.; Weiss, J.; J. Colloid Interface Sci. 2009, 334, 75.
27. Doroud, D.; Vatanara, A.; Zahedifard, F.; Gholami, E.; Vahabpour, R.; Rouholamini, N. A.; Rafati, S.; J. Pharm. Pharm. Sci. 2010, 13, 320.

28. Siddiqui, A.; Patwardhan, G. A.; Liu, Y. Y.; Nazzal, S.; Int. J. Pharm. 2010, 400, 251

29. Yang, L.; Geng, Y.; Li, H.; Zhang, Y.; You, J.; Chang, Y.; Pharmazie 2009, 64, 86.

30. Jain, S. K.; Chourasia, M. K.; Masuriha, R.; Soni, V.; Jain, A.; Jain, N. K.; Gupta, Y.; Drug Delivery 2005, 12, 207.

31. Igartua, M.; Saulnier, P.; Heurtault, B.; Pech, B.; Proust, J. E.; Pedraz, J. L.; Benoit, J. P.; Int. J. Pharm. 2002, 233, 149.

32. Hu, L.; Xing, Q.; Meng, J.; Shang, C.; AAPS Pharm. Sci. Tech. 2010 , 11,582 .

33. Hu, L.; Jia, H.; Luo, Z.; Liu, C.; Xing, Q.; Pharmazie 2010, 65, 110.

34. Attama, A. A.; Reichl, S.; Muller-Goymann, C. C.; Curr. Eye Res. 2009, 34, 698.

35. Muller, R. H.; Mader, K.; Gohla, S.; Eur. J. Pharm. Biopharm. 2000, 50, 161.

36. Eldem, T.; Speiser, P.; Hincal, A.; Pharm. Res. 1991, 8, 47.

37. Zur Muhlen, A.; Schwarz, C.; Mehnert, W.; Eur. J. Pharm. Biopharm. 1998, $45,149$.

38. Shegokar, R.; Singh, K. K.; Muller, R. H.; Int. J. Pharm. 2011, 416, 461.

39. Seo, M.; Gorelikoy, I.; Williams, R.; Matsuura, N.; Langmuir 2010, 26, 13855.

40. Yang, C. R.; Zhao, X. L.; Hu, H. Y.; Li, K. X.; Sun, X.; Li, L.; Chen, D. W.; Chem. Pharm. Bull. 2010, 58, 656.

41. Lippacher, A.; Mäder, K.; Int. J. Pharm. 2000, 196, 227.

42. Jenning, V.; Lippacher, A.; Gohla, S. H.; J. Microencapsulation 2002 , $19,1$.

43. Souto, E. B.; Müller, R. H.; J. Microencapsulation 2006, 23, 377.

44. Wang, D.; Zhao, P.; Cuia, F.; Li, X.; J. Pharm. Sci. Technol. 2007, 61, 110.

45. Gohla, S. H.; Dingler, A.; Pharmazie 2001, 56, 61.

46. Wang, W.; Zhao, X.; Hu, H.; Chen, D.; Gu, J.; Deng, Y.; Sun, J.; Drug Delivery 2010, 17, 114

47. Wang, D.; Wang, X.; Li, X.; Ye, L.; PDA J. Pharm. Sci. Technol. 2008, $62,56$.

48. Bhalekar, M. R.; Pokharkar, V.; Madgulkar, A.; Patil, N.; Patil, N.; AAPS Pharm. Sci. Tech. 2009, 10, 289.

49. Dingler, A.; Gohla, S.; J. Microencapsulation 2002, 19, 11.

50. You, J.; Wan, F.; De Cui, F.; Sun, Y.; Du, Y. Z.; Hu, F. Q.; Int. J. Pharm. 2007, 343, 270

51. Wan, F.; You, J.; Sun, Y.; Zhang, X. G.; Cui, F. D.; Du, Y. Z.; Yuan, H.; Hu, F. Q.; Int. J. Pharm. 2008, 359, 104.

52. Lippacher, A.; Müller, R. H.; Mäder, K.; Eur. J. Pharm. Biopharm. 2002, $53,155$.

53. Freitas, C.; Müller, R. H.; J. Microencapsulation 1999, 16, 59.

54. Souto, E. B.; Mehnert, W.; Müller, R. H.; J. Microencapsulation 2006, 23,417 .

55. Jenning, V.; Gohla, S.; Int. J. Pharm. 2000, 196, 219.

56. Kumar, V. V.; Chandrasekar, D.; Ramakrishna, S.; Kishan, V.; Rao, Y. M.; Diwan, P. V.; Int. J. Pharm. 2007, 335, 167.

57. Schubert, M. A.; Harms, M.; Muller-Goymann, C. C.; Eur. J. Pharm. Sci. 2006, 27, 226.

58. Attama, A. A.; Schicke, B. C.; Muller-Goymann, C. C.; Eur. J. Pharm. Biopharm. 2006, 64, 294.

59. Larsson, K.; Acta Chem. Scandinavia 1966, 20, 2255.

60. Freitas, C.; Müller, R. H.; Eur. J. Pharm. Biopharm. 1999, 47, 125.

61. Windbergs, M.; Strachan, C. J.; Kleinebudde, P.; AAPS Pharm. Sci. Tech. 2009, 10, 1224.

62. Bummer, P. M.; Crit. Rev. Ther. Drug Carrier Syst. 2004, $21,1$.

63. Müller, R. H.; Keck, C. M.; J. Biotechnol. 2004, 113, 151.

64. Rosenblatt, K. M.; Bunjes, H.; Mol. Pharm. 2009, 6, 105.

65. Bunjes, H.; Koch, M. H.; Westesen, K.; J. Pharm. Sci. 2003, 92, 1509. 
66. Bunjes, H.; Unruh, T.; Adv. Drug Delivery Rev. 2007, 59, 379.

67. Anantachaisilp, S.; Smith, S. M.; Treetong, A.; Pratontep, S.; Puttipipatkhachom, S.; Ruktanonchai, U. R.; Nanotechnology 2010, 21, 125102.

68. Paliwal, R.; Vaidya, B.; Khatri, K.; Goyal, A. K.; Mishra, N.; Mehta, A.; Vyas, S. P.; Nanomedicine 2009, 5, 184.

69. Ahlin, P.; Kristl, L.; Sentjurc, M.; Strancar, J.; Pecar, S.; Int. J. Pharm. 2000, 196, 241.

70. Siekmann, B.; Bunjes, H.; Koch, M. H.; Westesen, K.; Int. J. Pharm. 2002, 244, 33.

71. Yassin, A. E.; Anwer, M. K.; Mowafy, H. A.; El-Bagory, I. M.; Bayomi, M. A.; Alsarra, I. A.; Int. J. Med. Sci. 2010, 7, 398.

72. Liu, M.; Chen, J. H.; Dong, F. R.; Liu, Y.; Nan Fang Yi Ke Da Xue Xue Bao. 2008, 28, 700 .

73. Chen, H.; Chang, X.; Du, D.; Liu, W.; Liu, J.; Weng, T.; Yang, Y.; Xu, H.; Yang, X.; J. Controlled Release 2006, 110, 296.

74. Yang, S. C.; Lu, L. F.; Cai, Y.; Zhu, J. B.; Liang, B. W.; Yang, C. Z.; J. Controlled Release 1999, 59, 299.

75. Yang, Y.; Feng, J. F.; Zhang, H.; Luo, J. Y.; Zhongguo Zhong Yao Za Zhi. 2006, 31, 650 .

76. Schmidts, T.; Dobler, D.; Nissing, C.; Runkel, F.; J. Colloid Interface Sci. 2009, 338, 184; Jores, K.; Mehnert, W.; Drechsler, M.; Bunjes, H.; Johann, C.; Mader, K.; J. Controlled Release 2004, 95, 217.
77. Varia, J. K.; Dodiya, S. S.; Sawant, K .K.; Curr. Drug Delivery 2008, 5 , 64.

78. Varshosaz, J.; Tabbakhian, M.; Mohammadi, M. Y.; J. Liposome Res. 2010, 20, 286.

79. Zhu, R. R.; Qin, L. L.; Wang, M.; Wu, S. M.; Wang, S. L.; Zhang, R.; Liu, Z. X.; Sun, X. Y.; Yao, S. D.; Nanotechnology 2009, 20, 055702.

80. Yang, R.; Gao, R. C.; Li, F.; He, H.; Tang, X.; Drug Dev. Ind. Pharm. 2011, 37, 139.

81. Yang, R.; Gao, R. C.; Cai, C. F.; Xu, H.; Li, F.; He, H. B.; Tang, X.; Chem. Pharm. Bull. 2010, 58, 1195.

82. Tirnaksiz, F.; Kalsin, O.; J. Pharm. Pharm. Sci. 2005, 8, 299.

83. Gasco, M. R.; Priano, L.; Zara, G. P.; Prog. Brain Res. 2009, 180, 181.

84. Caboi, F.; Lazzari, P.; Pani, L.; Monduzzi, M.; Chem. Phys. Lipids 2005, $135,147$.

85. Mao, S. R.; Wang, Y. Z.; Ji, H. Y.; Bi, D. Z.; Yao Хие Хие Bao. 2003, 38, 624.

86. Kristl, J.; Volk, B.; Gasperlin, M.; Sentjurc, M.; Jurkovic, P.; Eur. J. Pharm. Sci. 2003, 19, 181.

87. Shah, K. A.; Joshi, M. D.; Patravale, V. B.; J. Biomed. Nanotechnol. 2009, 5, 396.

88. Marego, E.; Cavalli, R.; Caputo, O.; Rodriguez, L.; Gasco, M. R.; Int. J. Pharm. 2000, 205, 3. 